\title{
Incremental polynomial time dualization of quadratic functions and a subclass of degree- $k$ functions
}

\section{O. Ekin Karaşan}

Published online: 15 October 2009

(C) Springer Science+Business Media, LLC 2009

\begin{abstract}
We consider the problem of dualizing a Boolean function $f$ represented by a DNF. In its most general form, this problem is commonly believed not to be solvable by a quasi-polynomial total time algorithm. We show that if the input DNF is quadratic or is a special degree- $k$ DNF, then dualization turns out to be equivalent to hypergraph dualization in hypergraphs of bounded degree and hence it can be achieved in incremental polynomial time.
\end{abstract}

Keywords Boolean function · Dualization - Quadratic function · Degree- $k$ function · Hypergraph transversal · Polynomial total time algorithm

\section{Introduction}

Duality plays a fundamental role in many applications including reliability theory, hypergraph theory, game theory, and artificial intelligence. For an extensive list of applications, the reader is referred to Crama and Hammer (2009). However, it is well known that unless $\mathrm{P}=\mathrm{NP}$, there is no polynomial total time algorithm for the dualization problem of general Boolean functions. To this end, research on duality has been directed to special classes of Boolean functions.

The dualization problem, especially for positive DNFs (equivalently hypergraph dualization problem), has been widely investigated. (For a thorough survey see Bioch and Ibaraki (1995) and Eiter and Gottlob (1995)). In their seminal paper Fredman and Khachiyan (1996) give a dualization algorithm for positive DNFs which runs in incremental quasipolynomial time. Up to date, it is not known whether a dualization algorithm, whose running time is output polynomial, exists for positive Boolean functions. For a recent survey of the computational aspects on the dualization of positive DNFs, the interested reader is

In memory of Peter L. Hammer.

O.E. Karaşan (凶)

Department of Industrial Engineering, Bilkent University, Ankara, Turkey

e-mail: karasan@bilkent.edu.tr 
referred to Eiter et al. (2008). For degree- $k$ positive functions, dualization can be solved in incremental polynomial time (Boros et al. 1998; Eiter and Gottlob 1995). In the special case when $k=2$, even more efficient algorithms are possible (Johnson et al. 1988; Lawler et al. 1980; Makino and Uno 2004). Other efficiently solved special cases include 2-monotonic, threshold, regular, acyclic, and $\mathrm{O}(\log n)$-term functions (Boros et al. 1997; Crama 1987; Makino 2003; Makino and Ibaraki 1998; Peled and Simeone 1985, 1994). (For an extensive list of references, the reader is referred to Crama and Hammer (2009) and the survey Eiter et al. (2003)).

The literature of dualization results on general Boolean functions is far less extensive when compared with positive functions. Most of the research revolves around subclasses of Horn functions. In particular, dualization can be achieved in polynomial time for double Horn functions (Eiter et al. 1998), incremental polynomial time for submodular functions (Ekin 1997; Ekin et al. 1997), and in quasi-polynomial total time for disguised bidual Horn functions (Eiter et al. 2002) and Horn functions represented by Horn DNFs (Khardon 1995). The purpose of this paper is to extend the list of efficiently dualized classes to quadratic functions and a subclass of degree- $k$ functions.

Given a Boolean function $f$ represented by a quadratic DNF $\Phi\left(x_{1}, \ldots, x_{n}\right)$, we present in this paper a dualization algorithm which lists the dual prime implicants of $f$ in incremental polynomial time. The algorithm associates to $f$ a graph which can be constructed in time polynomial in $n$ and the size of $\Phi$, and which has the property that its minimal vertex covers are in one-to-one correspondence with the prime implicants of $f^{d}$. We then make use of one of the several algorithms existing in the literature (Johnson et al. 1988; Lawler et al. 1980) which generate all the maximal independent sets of a graph in incremental polynomial time. Furthermore, a similar construction is carried out for a subclass of degree- $k$ functions in which every prime implicant of cubic or higher degree conflicts with another in at most one variable. Associating a degree bounded hypergraph to such a function, we show that the prime implicants of the dual function are in one to one correspondence with the minimal transversals of this hypergraph and hence can be listed in output polynomial time due to algorithms in Boros et al. (1998) and in Eiter and Gottlob (1995).

The rest of the paper is organized as follows. In Sect. 2 we review the pertinent Boolean concepts and introduce the notation. In Sect. 3 we remind the reader of the earlier results on dualization of submodular Boolean functions and present our incremental polynomial dualization algorithm for quadratic functions. Section 4 extends the incremental polynomial time dualization algorithm to a subclass of degree- $k$ functions.

\section{Preliminaries on Boolean functions}

We assume that the reader is familiar with the basic concepts of Boolean algebra, and we only introduce here the notions and results that we explicitly use in this paper. We refer the interested reader to Crama and Hammer (2009).

A Boolean function $f\left(x_{1}, \ldots, x_{n}\right)$ is a mapping from $B^{n}$ into $B$, where $B=\{0,1\} . B^{n}$ is commonly referred to as the Boolean hypercube. If $f(\boldsymbol{x})=0$, the vector $\boldsymbol{x}$ is said to be a root of $f$. For every variable $x_{i}, \bar{x}_{i}$ denotes its complement, where $\overline{0}=1$ and $\overline{1}=0$. Let $\left\{x_{1}, \ldots, x_{n}\right\}$ be the set of variables, or positive literals and $\left\{\bar{x}_{1}, \ldots, \bar{x}_{n}\right\}$ be the set of Boolean complements of the variables, or negative literals. We shall sometimes denote $x_{i}$ by $x_{i}^{1}$ or $i^{1}$ for short and $\bar{x}_{i}$ by $x_{i}^{0}$ or $i^{0}$ for short. 
Let $P$ and $N$ be subsets of $\{1,2, \ldots, n\}$ satisfying $P \cap N=\emptyset$. A conjunction of literals of the form

$$
\bigwedge_{i \in P} x_{i} \bigwedge_{j \in N} \bar{x}_{j}
$$

is called a term, or an elementary conjunction. We usually use multiplicative notation to denote conjunctions $\wedge$. By convention, if $P=N=\emptyset$, the term is considered to be the constant 1 . The degree of a term is given by $|P \cup N|$. The term $T$ is called positive if $N=\emptyset$, negative if $P=\emptyset$, Horn if $|N| \leq 1$, linear if $|P \cup N|=1$ and quadratic if $|P \cup N|=2$.

The dual of a Boolean function $f(\boldsymbol{x})$ is defined as

$$
f^{d}(\boldsymbol{x})=\bar{f}(\overline{\boldsymbol{x}}),
$$

where $\overline{\boldsymbol{x}}=\left(\bar{x}_{1}, \bar{x}_{2}, \ldots, \bar{x}_{n}\right)$ is the complement of $\boldsymbol{x}$, and $\bar{f}$ is the complement of $f$, i.e., $\bar{f}(\boldsymbol{y})=1$ if and only if $f(\boldsymbol{y})=0$.

For two Boolean functions $f$ and $g$ we write $f \leq g$ if for every $0-1$ vector $\boldsymbol{x}$, $f\left(x_{1}, \ldots, x_{n}\right)=1$ implies $g\left(x_{1}, \ldots, x_{n}\right)=1$. An implicant of a Boolean function $f$ is a term $T$ such that $T \leq f$. We shall say that a term $T$ absorbs another term $T^{\prime}$, if $T \vee T^{\prime}=T$, i.e., if $T \geq T^{\prime}$ (e.g. the term $x \bar{y}$ absorbs the term $x \bar{y} z$ ). An implicant $T$ of a function is called prime if there is no other implicant of $f$ absorbing $T$.

A disjunctive normal form (DNF) is a Boolean formula of the form

$$
\Phi\left(x_{1}, \ldots, x_{n}\right)=\bigvee_{k=1}^{m}\left(\bigwedge_{i \in P_{k}} x_{i} \bigwedge_{j \in N_{k}} \bar{x}_{j}\right),
$$

where $P_{1}, \ldots, P_{m}, N_{1}, \ldots, N_{m}$ are subsets of the set $\{1, \ldots, n\}$ such that $P_{k} \cap N_{k}=\emptyset$ for $k=$ $1, \ldots, m$. It is well known that every Boolean function $f$ can be represented by a DNF, e.g., $\bigvee_{f\left(c_{1}, \ldots, c_{n}\right)=1} x_{1}^{c_{1}} x_{2}^{c_{2}} \ldots x_{n}^{c_{n}}$ and that this representation is not unique. A DNF representing a function $f$ is called prime if each term of the DNF is a prime implicant of the function. It is called minimum if there is no DNF representation of $f$ using fewer terms. It is called complete if it is the disjunction of all the prime implicants of $f$.

The focus of this paper is on the following dualization problem.

\section{DUALIZATION:}

Instance: A complete DNF representation of a Boolean function $f$.

Output: The complete DNF of $f^{d}$.

Note that in the special case of quadratic Boolean function dualization, one might equivalently assume that the given instance is a quadratic DNF since starting from any quadratic DNF representation of $f$ one can reach the complete DNF representation in polynomial time (Crama and Hammer 2009).

A Boolean function $f$ is called degenerate if there is an index $i$ and a fixed value $c \in$ $B$ such that $x_{i}=c$ for every root $\left(x_{1}, \ldots, x_{n}\right)$ of $f$; otherwise it is called nondegenerate. Obviously, a nonconstant Boolean function is degenerate if and only if it has a linear prime implicant.

Given a DNF $\Phi$, we denote by $|\Phi|$ and length $(\Phi)$ the number of terms and the number of literals in $\Phi$, respectively. The degree of $\Phi$ is the maximum degree of its terms. A Boolean function $f$ is called a degree- $k$ function if the degree of the complete DNF of $f$ is $k$.

Two terms are said to be orthogonal or to conflict in the variable $x_{i}$ if $x_{i}$ is a literal in one of them and $\bar{x}_{i}$ is a literal in the other. If the two terms conflict in exactly one variable, i.e., 
they have the form $x_{i} P$ and $\bar{x}_{i} Q$ and the elementary conjunctions $P$ and $Q$ have no conflict, their consensus is defined to be the term $P Q$. The consensus method applied to an arbitrary DNF $\Phi$ representation of a Boolean function $f$ performs the following operations as many times as possible:

Consensus: If there exist two terms of $\Phi$ having a consensus $T$ then replace the DNF $\Phi$ by the DNF $\Phi \vee T$.

Absorption: If a term $T^{\prime}$ of $\Phi$ absorbs a term $T^{\prime \prime}$ of $\Phi$, delete $T^{\prime \prime}$.

It is easy to notice that all the DNFs produced at every step of the consensus method represent the same function as the original DNF. The following result plays a central role in the theory and applications of Boolean functions (Blake 1937; Quine 1952):

Proposition 2.1 (Blake 1937; Quine 1952) The consensus method applied to an arbitrary DNF of a Boolean function $f$ results in the complete DNF of this function.

Within the scope of this study, quadratic and Horn functions play special roles. In particular, a quadratic function is a degree- 2 function. Equivalently, a Boolean function is quadratic if and only if it admits a quadratic DNF representation. On the other hand, a Boolean function is Horn if it has a DNF representation in which every term is Horn.

A vertex cover of a given graph $G=(V, E)$ is a subset $C \subseteq V$ such that $C$ meets all edges in $\mathrm{E}$ and a minimal vertex cover is simply a vertex cover which is minimal with respect to removal of vertices. Similarly, a minimal transversal of a hypergraph $\mathcal{H}=(V, \mathcal{E})$ is a set $S \subseteq V$ meeting every edge of $\mathcal{E}$ and being inclusionwise minimal.

The following notion will characterize the complexity of our dualization algorithms.

Definition 2.1 (Johnson et al. 1988; Lawler et al. 1980) An algorithm to enumerate items $a_{1}, a_{2}, \ldots, a_{p}$ is said to run in incremental polynomial time if

- it iterates the following procedure for $i=1,2, \ldots, p$ : output the $i$ th item $a_{i}$ from the knowledge of its input and items $a_{1}, a_{2}, \ldots, a_{i-1}$ generated so far, and

- the time required for the $i$ th iteration is polynomial in the input length and the sizes of $a_{1}, a_{2}, \ldots, a_{i-1}$.

\section{Dualization of quadratic Boolean functions}

Submodularity plays a prominent role in the study of set functions (pseudo-Boolean functions). The meaning of this property for the special class of Boolean functions has been thoroughly investigated in Ekin (1997) and in Ekin et al. (1997). Here we summarize the findings of Ekin et al. (1997) that will be pertinent in the forthcoming dualization results. In Ekin et al. (1997), a Boolean function is characterized as submodular if and only if it is both Horn and co-Horn, i.e., $f(\overline{\mathbf{x}})$ is Horn. Equivalently, it has been shown that all prime implicants of submodular functions are either linear or quadratic and pure-Horn, i.e., $|N|=1$ in every term of the form (1). Moreover, it has been shown that there is a one-to-one correspondence between submodular functions and partial preorders. This correspondence then led to a graph-theoretic characterization of all the prime implicants of the dual of a submodular function. The current study extends the dualization results on the submodular Boolean functions of Ekin (1997) and Ekin et al. (1997) to quadratic Boolean functions in general. 
In contrast to the numerous powerful characteristics of quadratic functions, it turns out that it is not easy to know whether an arbitrary given DNF represents a quadratic Boolean function. This is indicated by the following result.

Theorem 3.1 (Ekin et al. 2000) The recognition problem for the quadraticity property of a Boolean function given by a DNF is coNP-complete.

Hence, in this section, we restrict our attention to quadratic functions represented by quadratic DNFs. However, it is well known that the dualization of a quadratic Boolean function $f$ represented by a quadratic DNF (or even the complete DNF) may require exponential time in the size of the given DNF, the reason being the possibly exponential size of the DNFs of $f^{d}$. In particular, if $f$ is represented by the DNF

$$
\bigvee_{i=1}^{\frac{n}{2}} x_{2 i-1} \bar{x}_{2 i}
$$

where $n$ is even, then a minimum DNF of $f^{d}$ has $2^{\frac{n}{2}}$ terms.

In this section, we shall describe an incremental polynomial time algorithm for dualizing a nondegenerate quadratic function $f$ on $x_{1}, \ldots, x_{n}$. We remark that the nondegeneracy assumption is inessential and is made for convenience. Indeed, if $f=f_{0} \vee f_{1}$ where $f_{0}$ is linear, then each prime implicant of $f^{d}$ contains $f_{0}^{d}$, i.e. the conjunction of all the literals in $f_{0}$.

Let $\Phi$ given as (2), where $\left|P_{k} \cup N_{k}\right|=2$ for every $k=1, \ldots, m$, be the complete DNF representation of $f$. If $\Phi \equiv 1$, then $f^{d}=0$. We represent $\Phi$ defined on $\left\{x_{1}, \ldots, x_{n}\right\}$ by a graph $G_{f}=(W \cup \bar{W}, E)$, where $W=\{1, \ldots, n\}$ and $\bar{W}=\{\overline{1}, \ldots, \bar{n}\}$ unionwise define the vertex set of $G_{f}$. As for the edge set of this graph, we associate to each prime implicant $x_{i}^{\alpha} x_{j}^{\beta}$ for some $\alpha, \beta \in B$, an edge $\left\{i^{\alpha}, j^{\beta}\right\} \in E$.

By definition of the dual, the disjunction of all the prime implicants of $f^{d}$ is obtained from $\Phi$ by exchanging disjunctions and conjunctions, fully using distributivity, deleting terms involving both $x$ and $\bar{x}$, and performing absorptions. We thus have,

Lemma 3.1 The prime implicants of $f^{d}$ are in one-to-one correspondence with those minimal vertex covers of $G_{f}$ that do not contain both a vertex $i$ and its complement $\bar{i}$ for any $i \in W$.

\section{Proof Let}

$$
f=\Phi=\bigvee_{k=1}^{m}\left(\bigwedge_{i \in P_{k}} x_{i} \bigwedge_{j \in N_{k}} \bar{x}_{j}\right) .
$$

Then, by definition,

$$
f^{d}=\bigwedge_{k=1}^{m} \bigvee_{i \in P_{k}} x_{i} \bigvee_{j \in N_{k}} \bar{x}_{j}
$$

We note that a term $P=\prod_{j \in S} x_{j}^{\alpha_{j}}$ where $\alpha_{j} \in B$ for all $j \in S$ is a prime implicant of $f^{d}$ if and only if $P$ contains at least one literal from each clause (disjunction of literals) of the form $\bigvee_{i \in P_{k}} x_{i} \bigvee_{j \in N_{k}} \bar{x}_{j}$, where $\left|P_{k} \cup N_{k}\right|=2 \forall k$, and $S$ is minimal with this property (see 
Crama and Hammer 2009). Since every minimal vertex cover of $G_{f}$ not containing both a vertex $i$ and its complement $\bar{i}$ satisfies this property, we get the desired result.

The definition of equivalence given for submodular functions in Ekin (1997) and in Ekin et al. (1997) has to be revised for quadratic functions. More formally,

Definition 3.1 Two literals $x_{i}^{\alpha}$ and $x_{j}^{\beta}$ for $\alpha, \beta \in B$ are called equivalent if both $x_{i}^{\alpha} x_{j}^{\bar{\beta}}$ and $x_{i}^{\bar{\alpha}} x_{j}^{\beta}$ are prime implicants of $f$.

It is easy to see that if $x_{i}^{\alpha}$ and $x_{j}^{\beta}$ are equivalent, then by definition $x_{i}^{\bar{\alpha}}$ and $x_{j}^{\bar{\beta}}$ are also equivalent and that equivalence is a transitive relationship. If $x_{i}^{\alpha}$ and $x_{j}^{\beta}$ are equivalent, we shall shortly state that $i^{\alpha}$ and $j^{\beta}$ are equivalent.

Lemma 3.2 Let $C$ be a minimal vertex cover of $G_{f}$ that does not contain both a vertex and its negation. If $i^{\alpha}$ and $j^{\beta}$ are equivalent, then $C$ either contains both $i^{\alpha}$ and $j^{\beta}$ or both $i^{\bar{\alpha}}$ and $j^{\bar{\beta}}$.

Proof If $i^{\alpha} \in C$, then $i^{\bar{\alpha}} \notin C$ by our assumption on $C$. Also, the equivalence of $i^{\alpha}$ and $j^{\beta}$ implies that $\left\{i^{\bar{\alpha}}, j^{\beta}\right\} \in E$. Therefore, in order to cover this edge, $C$ must contain $j^{\beta}$. In a similar way, one can conclude that if $i^{\bar{\alpha}}$ belongs to $C$ then so does $j^{\bar{\beta}}$. Finally, since both $\left\{i^{\alpha}, j^{\bar{\beta}}\right\}$ and $\left\{i^{\bar{\alpha}}, j^{\beta}\right\}$ are edges of $G_{f}$, any vertex cover satisfying the hypothesis must contain either $i^{\alpha}$ or $i^{\bar{\alpha}}$.

Let $G_{f}^{*}=\left(V^{*}, E^{*}\right)$ be the graph obtained from $G_{f}$ in the following way. We identify all vertices in an equivalence class of $W \cup \bar{W}$ into a single vertex in $V^{*}$. With this identification, the equivalence classes come in pairs. In particular, if $\mathcal{I}=\left\{i_{1}^{\alpha_{1}}, i_{2}^{\alpha_{2}}, \ldots, i_{k}^{\alpha_{k}}\right\}$ is an equivalence class corresponding to node $I$ of $V^{*}$, then $\overline{\mathcal{I}}=\left\{i_{1}^{\bar{\alpha}_{1}}, i_{2}^{\bar{\alpha}_{2}}, \ldots, i_{k}^{\bar{\alpha}_{k}}\right\}$ is also an equivalence class corresponding to node $\bar{I}$ of $V^{*}$. Note that an equivalence class may happen to consist of a single vertex.

By definition, in $G_{f}^{*}$, there is an edge between two nodes $I^{\alpha}$ and $J^{\beta}$ respectively corresponding to equivalence classes $\mathcal{I}^{\alpha}$ and $\mathcal{J}^{\beta}$ if and only if $\left\{i^{a}, j^{b}\right\}$ is an edge of $G_{f}$ for some $i^{a} \in \mathcal{I}^{\alpha}$, and $j^{b} \in \mathcal{J}^{\beta}$ where $a, b, \alpha, \beta \in B$. With the definition of equivalence classes, we must have $\{I, \bar{I}\} \in E^{*}$ for every equivalence class $\mathcal{I}$ such that $|\mathcal{I}| \geq 2$. As it has already been established in Lemma 3.2, if $C$ is a minimal vertex cover of $G_{f}$ that does not contain both a vertex and its negation, then for every equivalence class $\mathcal{I}$ such that $|\mathcal{I}| \geq 2, C$ must either contain all elements in $\mathcal{I}$ or all elements in $\overline{\mathcal{I}}$. Hence, a minimal vertex cover of $G_{f}$ that does not contain both a vertex and its negation corresponds to a minimal vertex cover of $G_{f}^{*}$ that does not contain a vertex and its negation. In fact, we shall assert a stronger statement with Lemma 3.5 but prior to that we first make two simple observations which together guarantee that no information is lost during the process of shrinking the equivalent vertices into single ones.

Lemma 3.3 No edge of $G_{f}$ joins a vertex to an equivalent vertex.

Proof Assume to the contrary that $i^{\alpha}$ and $j^{\beta}$ are two equivalent literals for which $\left\{i^{\alpha}, j^{\beta}\right\} \in E$. By definition of equivalence in Definition 3.1, and the construction of $G_{f}$, $x_{i}^{\alpha} x_{j}^{\bar{\beta}}, x_{i}^{\bar{\alpha}} x_{j}^{\beta}$, and $x_{i}^{\alpha} x_{j}^{\beta}$ must all be prime implicants of $\Phi$ which violates the definition of primality. 
Lemma 3.4 If $\left\{I^{\alpha}, J^{\beta}\right\} \in E^{*}$, then $x_{i}^{a} x_{j}^{b}$ is a prime implicant of $f \forall i^{a} \in \mathcal{I}^{\alpha}$ and $\forall j^{b} \in \mathcal{J}^{\beta}$ such that $i \neq j$.

Proof The result trivially follows from the consensus procedure and the definition of equivalence. Indeed, let $\left|\mathcal{I}^{\alpha}\right| \geq 2$ and say $i_{1}^{a_{1}}, i_{2}^{a_{2}} \in \mathcal{I}^{\alpha}$ and $j^{b} \in \mathcal{J}^{\beta}$. Now, if $x_{i_{1}}^{a_{1}} x_{j}^{b}$ is a prime implicant of $f$ then so is $x_{i_{2}}^{a_{2}} x_{j}^{b}$ since by the equivalence of $x_{i_{1}}^{a_{1}}$ and $x_{i_{2}}^{a_{2}}, x_{i_{1}}^{a_{1}} x_{i_{2}}^{a_{2}}$ is a prime implicant of $f$ and its consensus with $x_{i_{1}}^{a_{1}} x_{j}^{b}$ yields $x_{i_{2}}^{a_{2}} x_{j}^{b}$.

Lemma 3.5 No minimal vertex cover of $G_{f}^{*}$ contains both a vertex and its negation.

Proof Let $M$ be a minimal vertex cover of $G_{f}^{*}$. Barring trivial cases, we may assume that $|M| \geq 4$. Let us suppose that $M$ contains both a vertex $I$ and its negation $\bar{I}$. By the minimality of $M$ it follows that there exists a vertex $J^{\alpha} \notin M$ such that $\left\{I, J^{\alpha}\right\}$ is an edge of $G_{f}^{*}$. Similarly, there exists a vertex $K^{\beta} \notin M$ such that $\left\{\bar{I}, K^{\beta}\right\}$ is an edge of $G_{f}^{*}$. Note that it is not possible to have $J^{\alpha}=K^{\beta}$. Assume to the contrary that $I$ and $\bar{I}$ (where $\mathcal{I}$ could be singleton) correspond to an equivalence class and its negation, respectively, having a common neighbor $J^{\alpha}$ (where $\left|\mathcal{J}^{\alpha}\right|$ could be 1 ) in $G_{f}^{*}$. Since $\left\{I, J^{\alpha}\right\} \in E^{*}$ and $\left\{\bar{I}, J^{\alpha}\right\} \in E^{*}$, by the previous lemma, $x_{i}^{a} x_{j}^{b}$ and $x_{i}^{\bar{a}} x_{j}^{b}$ must be prime implicants of $f$ for all $i^{a} \in \mathcal{I}$ and $j^{b} \in \mathcal{J}^{\beta}$. However, this is in contradiction with the primality of these terms. So, $J^{\alpha} \neq K^{\beta}$. However, in this case $\left\{J^{\alpha}, K^{\beta}\right\}$ is an edge of $G_{f}^{*}$ which is not covered by $M$. Indeed, if both edges $\left\{I, J^{\alpha}\right\},\left\{\bar{I}, K^{\beta}\right\}$ are present, it means that there exist $i^{a} \in \mathcal{I}, j^{b} \in \mathcal{J}^{\alpha}$, and $k^{c} \in \mathcal{K}^{\beta}$ such that both $x_{i}^{a} x_{j}^{b}$ and $x_{i}^{\bar{a}} x_{k}^{c}$ are prime implicants of $f$, in which case their consensus i.e. $x_{j}^{b} x_{k}^{c}$ must also be a prime implicant of $f$ (Note that $J^{\bar{\alpha}}=K^{\beta}$ is not a possibility since it would mean $\mathcal{I} \cup \mathcal{J}^{\bar{\alpha}}$ is contained in some equivalence class).

We conclude that the minimal vertex covers of $G_{f}$ that do not contain a vertex and its negation (the prime implicants of $f^{d}$ ) correspond precisely to the minimal vertex covers of $G_{f}^{*}$.

There exist several algorithms in the literature for generating all maximal independent sets of a graph $G=(V, E)$ in incremental polynomial time (Johnson et al. 1988; Lawler et al. 1980). Since the maximal independent sets are precisely the complements of the minimal vertex covers, we have established the following result.

Theorem 3.2 Dualization of quadratic functions can be performed in incremental polynomial time.

We shall summarize the dualization algorithm by an example.

Example 3.1 Let the Boolean function $f$ be given by the quadratic DNF

$$
x_{1} \vee \bar{x}_{1} x_{2} \vee \bar{x}_{3} \bar{x}_{4} \vee x_{4} x_{5} \vee x_{3} \bar{x}_{5} \vee x_{4} \bar{x}_{6} \vee x_{5} \bar{x}_{7} \vee x_{6} x_{8}
$$

- Step 1: Find the complete DNF representation $\Phi$ of $f$. If $f=1$ then $f^{d}=0$ and the algorithm stops. In this example:

$$
\begin{gathered}
\Phi=x_{1} \vee x_{2} \vee \bar{x}_{3} \bar{x}_{4} \vee \bar{x}_{3} x_{5} \vee x_{3} x_{4} \vee x_{4} x_{5} \vee x_{3} \bar{x}_{5} \vee \bar{x}_{4} \bar{x}_{5} \vee \bar{x}_{3} \bar{x}_{6} \vee x_{4} \bar{x}_{6} \vee \bar{x}_{5} \bar{x}_{6} \\
\vee x_{3} \bar{x}_{7} \vee \bar{x}_{4} \bar{x}_{7} \vee x_{5} \bar{x}_{7} \vee \bar{x}_{6} \bar{x}_{7} \vee x_{6} x_{8} \vee \bar{x}_{7} x_{8} \vee \bar{x}_{3} x_{8} \vee x_{4} x_{8} \vee \bar{x}_{5} x_{8} .
\end{gathered}
$$


Fig. $1 G_{f}^{*}$

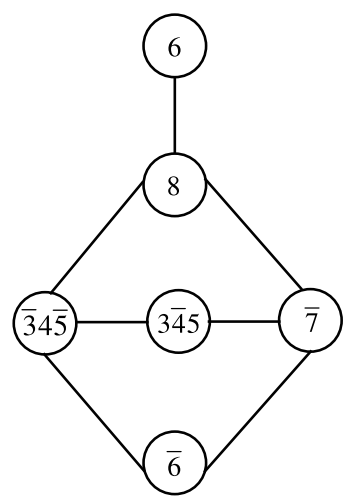

- Step 2: Identify all the equivalence classes. In this example, literals $\bar{x}_{3}, x_{4}$, and $\bar{x}_{5}$ are equivalent.

- Step 3: Construct $G_{f}^{*}$ (see Fig. 1). Note that the vertices corresponding to literals 7 and $\overline{8}$ can be eliminated from $G_{f}^{*}$ without any loss since they will not appear in any prime implicant of $f^{d}$.

- Step 4: Find all the maximal independent sets of $G_{f}^{*}$. Each maximal independent set corresponds to a prime implicant of $f^{d}$. In our example, we have 3 maximal independent sets.

$$
\begin{aligned}
& \text { Maximal Independent Set Corresponding prime implicant of } f^{d} \\
& \begin{array}{ll}
\overline{3} 4 \overline{5}, 6, \overline{7} & x_{1} x_{2} x_{3} \bar{x}_{4} x_{5} \bar{x}_{6} x_{8} \\
3 \overline{4} 5, \overline{6}, 6 & x_{1} x_{2} \bar{x}_{3} x_{4} \bar{x}_{5} \bar{x}_{7} x_{8} \\
3 \overline{4} 5, \overline{6}, 8 & x_{1} x_{2} \bar{x}_{3} x_{4} \bar{x}_{5} x_{6} \bar{x}_{7}
\end{array}
\end{aligned}
$$

where we have appended the conjunction of linear terms in $\Phi$ to each prime implicant.

\section{Dualization of a special class of degree- $k$ functions}

In this section, we extend the results on dualization of quadratic functions to a special class of Boolean functions as defined below:

Definition 4.1 A Boolean function of degree $k$ such that each prime implicant of degree $\geq 3$ conflicts with another prime implicant in at most one literal is called a mild degree- $k$ function.

In particular, mild degree-2 functions are quadratic functions. Any positive Boolean function of degree $k$ or an acyclic Horn function (Hammer and Kogan 1995) of degree $k$ is a mild degree- $k$ function. However, mild degree- $k$ functions are not limited to quadratic or Horn classes. The following is a mild degree- 4 function on 11 variables which is not q-Horn (a class introduced in Boros et al. 1990 which properly generalizes quadratic and Horn functions).

$$
\begin{aligned}
\Phi= & x_{4} x_{5} \vee x_{4} x_{6} \vee x_{4} x_{7} \vee x_{5} x_{6} \vee x_{5} x_{7} \vee x_{6} x_{7} \vee x_{1} \bar{x}_{2} x_{4} x_{8} \vee x_{1} x_{2} x_{5} x_{9} \vee \bar{x}_{1} \bar{x}_{3} x_{6} x_{10} \\
& \vee \bar{x}_{1} x_{3} x_{7} x_{11} \vee \bar{x}_{8} \bar{x}_{9} \bar{x}_{10} \bar{x}_{11} \vee x_{4} \bar{x}_{9} \bar{x}_{10} \bar{x}_{11} \vee x_{5} \bar{x}_{8} \bar{x}_{10} \bar{x}_{11} \vee x_{7} \bar{x}_{8} \bar{x}_{9} \bar{x}_{10} \vee x_{6} \bar{x}_{8} \bar{x}_{9} \bar{x}_{11} .
\end{aligned}
$$


Let $\Phi$ be the complete DNF representation of a mild degree- $k$ function $f$. Say,

$$
\Phi\left(x_{1}, \ldots, x_{n}\right)=\bigvee_{k=1}^{m}\left(\bigwedge_{i \in P_{k}} x_{i} \bigwedge_{j \in N_{k}} \bar{x}_{j}\right),
$$

Without loss of generality, we keep assuming that $\Phi$ does not have the constant 1 or linear terms as prime implicants. Following a similar construction as for the graph $G_{f}$ in Sect. 3, let $\mathcal{H}_{f}=(W \cup \bar{W}, \mathcal{E})$ be the hypergraph associated with this function. In particular, $W=$ $\{1, \ldots, n\}, \bar{W}=\{\overline{1}, \ldots, \bar{n}\}$, and $\mathcal{E}=\left\{E_{1}, \ldots, E_{m}\right\}$, where $E_{k}=\left\{i: i \in P_{k}\right\} \cup\left\{\bar{j}: j \in N_{k}\right\}$. In other words, the vertices of this hypergraph are the literals appearing in $\Phi$ and the hyperedges correspond to the terms in $\Phi$.

Let us recall Definition 3.1 on the equivalence of literals. Proceeding as we did in Sect. 4, let $\mathcal{H}_{f}^{*}=\left(V^{*}, \mathcal{E}^{*}\right)$ be the hypergraph obtained from $\mathcal{H}_{f}$ where equivalence classes and their negations from $W \cup \bar{W}$ are respectively identified as single vertices in $V^{*}$. One can easily attain parallel results to those stated in Lemmas 3.1-3.4 for mild degree- $k$ functions as follows:

\section{Lemma 4.1}

(1) The prime implicants of $f^{d}$ are in one-to-one correspondence with those minimal transversals of $H_{f}$ that do not contain both a vertex $i$ and its complement $\bar{i}$ for any $i \in W$.

(2) Let $S$ be a minimal transversal of $\mathcal{H}_{f}$ that does not contain both a vertex and its negation. Then, for any equivalence class $\mathcal{I}$ such that $|\mathcal{I}| \geq 2, S$ either contains $\mathcal{I}$ or its negation in its entirety.

(3) No hyperedge of $\mathcal{H}_{f}$ contains two equivalent literals.

(4) If $x^{\alpha} P$ where $P$ is a conjunction of literals, is a prime implicant of $f$, then so is $y^{\beta} P$ for every $y^{\beta}$ which is equivalent to $x^{\alpha}$.

Proof The proofs are basic extensions of those stated for Lemmas 3.1-3.4 and therefore are omitted here.

Lemma 4.2 If there exists a prime implicant $P$ in $\Phi$ of degree $\geq 3$ which conflicts with another prime implicant $Q$ in $\Phi$, then the consensus of $P$ and $Q$ exists and is absorbed by another prime implicant $T$ in $\Phi$.

Proof Since $P$ and $Q$ conflict, and since $P$ is not quadratic, they must conflict in exactly one variable. In other words, $P$ is of the form $x^{\alpha} P^{\prime}$ and $Q$ is of the form $x^{\bar{\alpha}} Q^{\prime}$ where $P^{\prime}$ and $Q^{\prime}$ are elementary conjunctions that do not conflict. Then their consensus $P^{\prime} Q^{\prime}$ is an implicant of the underlying function which must be absorbed by a prime implicant $T$ in $\Phi$ since $\Phi$ is complete.

Lemma 4.3 No minimal vertex cover of $\mathcal{H}_{f}^{*}$ contains both a vertex and its negation.

Proof Assume to the contrary that $M$ is a vertex cover of cardinality at least 5 containing both $I$ and $\bar{I}$. By the minimality of $M$, there exist $E^{\prime} \in \mathcal{E}^{*}$ where $I \in E^{\prime}$ and $E^{\prime \prime} \in \mathcal{E}^{*}$ where $\bar{I} \in E^{\prime \prime}$ and no vertex from $\left(E^{\prime} \backslash I\right) \cup\left(E^{\prime \prime} \backslash \bar{I}\right)$ belongs to $M$. Repeating the arguments in the proof of Lemma 3.5, it is not possible to have $\left|E^{\prime}\right|=\left|E^{\prime \prime}\right|=2$. Without loss of generality, we may assume that $\left|E^{\prime}\right| \geq 3$. Note that it is not possible to have $J^{\alpha} \in E^{\prime} \backslash I$ and $J^{\bar{\alpha}} \in$ 
$E^{\prime \prime} \backslash \bar{I}$ since this would violate the fact that two prime implicants of $f$ (one corresponding to hyperedge $E^{\prime}$ and the other corresponding to hyperedge $E^{\prime \prime}$ ) one of which is not quadratic (any one of the possible prime implicants corresponding to hyperedge $E^{\prime}$ ) conflict in at most one literal. Therefore, due to Lemma 4.2, in $\mathcal{E}^{*}$ there must exist an edge $E^{\prime \prime \prime}$ such that $E^{\prime \prime \prime} \subseteq\left(E^{\prime} \backslash I\right) \cup\left(E^{\prime \prime} \backslash \bar{I}\right)$. However, this edge is not covered by $M$.

Using the hypergraph dualization results of Boros et al. (1998) and Eiter and Gottlob (1995) for bounded degree hypergraphs (those for which each edge has a bounded cardinality) along with Lemma 4.3, we have:

Theorem 4.1 Dualization of mild degree-k functions given in complete DNF form can be performed in incremental polynomial time.

Acknowledgements The author is grateful to an anonymous referee whose detailed comments led to an improved presentation of the paper. The author also acknowledges the support from the Turkish Academy of Science.

\section{References}

Blake, A. (1937). Canonical expressions in Boolean algebras. Dissertation, Department of Mathematics, University of Chicago. Published by University of Chicago Libraries, 1938.

Bioch, J. C., \& Ibaraki, T. (1995). Complexity of identification and dualization of positive Boolean functions. Information and Computation, 123, 50-63.

Boros, E., Crama, Y., \& Hammer, P. L. (1990). Polynomial-time inference of all valid implications for Horn and related formulae. Annals of Mathematics and Artificial Intelligence, 1, 21-31.

Boros, E., Hammer, P. L., Ibaraki, T., \& Kawakami, K. (1997). Polynomial-time recognition of 2-monotonic positive Boolean functions given by an oracle. SIAM Journal on Computing, 26, 93-109.

Boros, E., Gurvich, V., \& Hammer, P. L. (1998). Dual subimplicants of positive Boolean functions. Optimization Methods and Software, 10, 147-156.

Crama, Y. (1987). Dualization of regular Boolean functions. Discrete Applied Mathematics, 16, 79-85.

Crama, Y., \& Hammer, P. L. et al. (2009, in press). Boolean functions theory algorithms and applications.

Eiter, T., \& Gottlob, G. (1995). Identifying the minimal transversals of a hypergraph and related problems. SIAM Journal on Computing, 24, 1278-1304.

Eiter, T., Ibaraki, T., \& Makino, K. (1998). Double Horn functions. Information and Computation, 144, 155190.

Eiter, T., Ibaraki, T., \& Makino, K. (2002). Recognition and dualization of disguised bidual Horn functions. Information Processing Letters, 82, 283-291.

Eiter, T., Gottlob, G., \& Makino, K. (2003). New results on monotone dualization and generating hypergraph transversals. SIAM Journal on Computing, 32(2), 514-537.

Eiter, T., Makino, K., \& Gottlob, G. (2008). Computational aspects of monotone dualization: A brief survey. Discrete Applied Mathematics, 156, 2035-2049.

Ekin, O. (1997). Special classes of Boolean functions. Ph.D. Thesis, Rutgers University, Piscataway, USA.

Ekin, O., Hammer, P. L., \& Peled, U. N. (1997). Horn functions and submodular Boolean functions. Discrete Applied Mathematics, 175, 257-270.

Ekin, O., Foldes, S., Hammer, P. L., \& Hellerstein, L. (2000). Equational characterizations of Boolean function classes. Discrete Mathematics, 211, 27-51.

Fredman, M., \& Khachiyan, L. (1996). On the complexity of dualization of monotone disjunctive normal forms. Journal of Algorithms, 21, 618-628.

Hammer, P. L., \& Kogan, A. (1995). Quasi-acyclic propositional Horn knowledge bases. IEEE Transaction on Knowledge and Data Engineering, 7(5), 751-762.

Johnson, D. S., Yannakakis, M., \& Papadimitriou, C. (1988). On generating all maximal independent sets. Information Processing Letters, 27, 119-123.

Khardon, R. (1995). Translating between Horn representations and their characteristic nodels. Journal of Artificial Intelligence Research, 3, 349-372.

Lawler, E. L., Lenstra, J. K., \& Rinooy Kan, H. G. (1980). Generating all maximal independent sets: NPhardness and polynomial-time algorithms. SIAM Journal on Computing, 9, 558-565. 
Makino, K. (2003). Efficient dualization of $\mathrm{O}(\log$ n)-term monotone disjunctive normal forms. Discrete Applied Mathematics, 126, 305-312.

Makino, K., \& Ibaraki, T. (1998). A fast and simple algorithm for identifying 2-monotonic positive Boolean functions. Journal of Algorithms, 26, 291-305.

Makino, K., \& Uno, T. (2004). New algorithms for enumerating all maximal cliques. In Lecture notes in computer science (Vol. 3111, pp. 260-272). Berlin: Springer.

Peled, U. N., \& Simeone, B. (1985). Polynomial-time algorithms for regular set-covering and threshold synthesis. Discrete Applied Mathematics, 12, 57-69.

Peled, U. N., \& Simeone, B. (1994). An $O(\mathrm{~nm})$-time algorithm for computing the dual of a regular Boolean function. Discrete Applied Mathematics, 49, 309-323.

Quine, W. V. (1952). The problem of simplifying truth functions. American Mathematical Monthly, 59, 521531 . 